\title{
Parahoric Restriction for GSp(4)
}

\author{
Mirko Rösner
}

\begin{abstract}
Parahoric restriction is the parahoric analogue of Jacquet's functor. Fix an arbitrary parahoric subgroup of the group $\operatorname{GSp}(4, F)$ of symplectic similitudes of genus two over a local number field $F / \mathbb{Q}_{p}$. We determine the parahoric restriction of the non-cuspidal irreducible smooth representations in terms of explicit character values.
\end{abstract}

\section{Introduction}

For a reductive connected group $\mathbf{G}$ over a local number field $F / \mathbb{Q}_{p}$, with group of $F$ valued points $G=\mathbf{G}(F)$, fix a compact parahoric subgroup $\mathscr{P} \subseteq G$ with pro-unipotent radical $\mathscr{P}^{+}$. The parahoric restriction functor between categories of admissible representations

$$
\mathbf{r}_{\mathscr{P}}: \operatorname{Rep}(G) \rightarrow \operatorname{Rep}\left(\mathscr{P} / \mathscr{P}^{+}\right), \quad(\rho, V) \mapsto\left(\left.\rho\right|_{\mathscr{P}}, V^{\mathscr{P}+}\right) .
$$

is the parahoric analogue of Jacquet's functor of parabolic restriction. It assigns to an admissible representation $(\rho, V)$ of $G$ the action of the Levi quotient $\mathscr{P} / \mathscr{P}^{+}$on the space of invariants under $\mathscr{P}^{+}$. The functor is exact and factors over semisimplification, so it is sufficient to study irreducible admissible representations.

For the group $\mathbf{G}=\mathrm{GSp}(4)$ of symplectic similitudes of genus two, we determine the parahoric restriction of non-cuspidal irreducible admissible representations in terms of explicit character values for the finite Levi quotient $\mathscr{P} / \mathscr{P}^{+}$. This has applications in the theory of Siegel modular forms of genus two, invariant under principal congruence subgroups of squarefree level.

\subsection{Main result}

For a proper parabolic subgroup of $G=\operatorname{GSp}(4, F)$ fix a cuspidal irreducible admissible complex linear representation $\sigma$ of its Levi quotient. Let $\rho$ be a subquotient of the normalized parabolic induction of $\sigma$ to $G$. Then $\rho$ is non-cuspidal and every non-cuspidal irreducible admissible representation of $G$ arises this way. 
The conjugacy classes of parahoric subgroups in $G$ are represented by the standard parahoric subgroups $\mathscr{B}, \mathscr{P}, \mathscr{Q}, \mathscr{K}, \mathscr{J}$ described below. We determine the parahoric restriction of $\rho$ with respect to each of these standard parahoric subgroups. If $\sigma$ has positive depth, then the parahoric restriction of $\rho$ is zero, because the depths of $\sigma$ and $\rho$ coincide [5, 5.2].

Theorem 1.1. Suppose $\sigma$ has depth zero. i) The parahoric restriction of $\rho$ with respect to the standard hyperspecial parahoric subgroup $\mathscr{K}$ is given by Table 3$]_{1}^{1}$

ii) The parahoric restriction of $\rho$ with respect to the standard Iwahori $\mathscr{B}$, standard Siegel parahoric $\mathscr{P}$ and standard Klingen parahoric $\mathscr{Q}$ is given by Table 4.

iii) The parahoric restriction of $\rho$ with respect to the standard paramodular group $\mathscr{J}$ is given by Table 5 .

The proof is in Section 3, the tables are in Section 4. By character theory, we can determine invariants for every subgroup of the Levi quotients. For the full Levi we obtain parahori-spherical vectors ${ }^{2}$

Corollary 1.2. The parahori-spherical irreducible admissible representations of $G$ are exactly the subquotients of the unramified principal series $\mu_{1} \times \mu_{2} \rtimes \mu_{0}$ for unramified characters $\mu_{1}, \mu_{2}, \mu_{0}$ of $F^{\times}$. The dimension of parahori-spherical vectors is given by Table 1 .

Proof. For non-cuspidal irreducible admissible representations, the dimension of parahorispherical vectors equals the multiplicity of the trivial representation in the parahoric restriction. Cuspidal representations are never parahori-spherical [1, 4.7], [5, 6.11].

\section{Preliminaries}

Fix a nonarchimedean local number field $F$ with finite residue field $\mathfrak{o} / \mathfrak{p} \cong \mathbb{F}_{q}$ of order $q$. The valuation character $\nu=|\cdot|$ of $F^{\times}$is normalized such that $|\varpi|=q^{-1}$ for a uniformizing element $\varpi \in \mathfrak{p}$.

\subsection{The parahoric restriction functor}

Let $G$ be the group of $F$-rational points of a connected reductive linear algebraic group over a non-archimedean local number field $F$. Let $\mathscr{P} \subseteq G$ be a parahoric subgroup with Levi decomposition

$$
1 \rightarrow \mathscr{P}^{+} \rightarrow \mathscr{P} \rightarrow \underline{\mathscr{P}} \rightarrow 1
$$

\footnotetext{
${ }^{1}$ Partial results in this case have been obtained before by Breeding [2].

${ }^{2}$ This coincides with previous work by Roberts and Schmidt [6, Table A.15].
} 
Table 1: Dimension of parahori-spherical vectors for unramified characters $\mu_{1}, \mu_{2}, \mu_{0}, \xi$.

\begin{tabular}{lcccccc}
\hline type & $\rho$ of $\mathrm{GSp}(4, F)$ & $\operatorname{dim} \rho^{\mathscr{K}}$ & $\operatorname{dim} \rho^{\mathscr{J}}$ & $\operatorname{dim} \rho^{\mathscr{P}}$ & $\operatorname{dim} \rho^{\mathscr{Q}}$ & $\operatorname{dim} \rho^{\mathscr{B}}$ \\
\hline I & $\mu_{1} \times \mu_{2} \rtimes \mu_{0}$ & 1 & 2 & 4 & 4 & 8 \\
IIa & $\mu_{1} \mathrm{St} \rtimes \mu_{0}$ & 0 & 1 & 1 & 2 & 4 \\
IIb & $\mu_{1} \mathbf{1} \rtimes \mu_{0}$ & 1 & 1 & 3 & 2 & 4 \\
IIIa & $\mu_{1} \rtimes \mu_{0} \mathrm{St}$ & 0 & 0 & 2 & 1 & 4 \\
IIIb & $\mu_{1} \rtimes \mu_{0} \mathbf{1}$ & 1 & 2 & 2 & 3 & 4 \\
IVa & $\mu_{0} \mathrm{St} t_{\mathrm{GSp}}(4, F)$ & 0 & 0 & 0 & 0 & 1 \\
IVb & $L\left(\nu^{2}, \nu^{-1} \mu_{0} \mathrm{St}\right)$ & 0 & 0 & 2 & 1 & 3 \\
IVc & $L\left(\nu^{3 / 2} \mathrm{St}, \nu^{-3 / 2} \mu_{0}\right)$ & 0 & 1 & 1 & 2 & 3 \\
IVd & $\mu_{0} \mathbf{1}_{\mathrm{GSp}(4, F)}$ & 1 & 1 & 1 & 1 & 1 \\
Va & $\delta\left(\left[\xi_{u}, \nu \xi_{u}\right], \nu^{-1 / 2} \mu_{0}\right)$ & 0 & 0 & 0 & 1 & 2 \\
Vb & $L\left(\nu^{1 / 2} \xi_{u} \mathrm{St}, \nu^{-1 / 2} \mu_{0}\right)$ & 0 & 1 & 1 & 1 & 2 \\
Vc & $L\left(\nu^{1 / 2} \xi_{u} \mathrm{St}, \nu^{-1 / 2} \xi_{u} \mu_{0}\right)$ & 0 & 1 & 1 & 1 & 2 \\
Vd & $L\left(\nu \xi_{u}, \xi_{u} \rtimes \nu^{-1 / 2} \mu_{0}\right)$ & 1 & 0 & 2 & 1 & 2 \\
VIa & $\tau\left(S, \nu^{-1 / 2} \mu_{0}\right)$ & 0 & 0 & 1 & 1 & 3 \\
VIb & $\tau\left(T, \nu^{-1 / 2} \mu_{0}\right)$ & 0 & 0 & 1 & 0 & 1 \\
VIc & $L\left(\nu^{1 / 2} \mathrm{St}, \nu^{-1 / 2} \mu_{0}\right)$ & 0 & 1 & 0 & 1 & 1 \\
VId & $L\left(\nu, 1_{F} \rtimes \nu^{-1 / 2} \mu_{0}\right)$ & 1 & 1 & 2 & 2 & 3 \\
\hline
\end{tabular}

where $\mathscr{P}$ is a finite reductive group over the residue field. For an admissible complex representation $\pi: G \rightarrow \operatorname{Aut}(V)$ on a complex vector space $V$, the action of $\mathscr{P}$ preserves the subspace $V^{\mathscr{P}^{+}}$of $\mathscr{P}^{+}$-invariants in $V$. This defines a representation $\left(\left.\rho\right|_{\mathscr{P}}, V^{\mathscr{P}^{+}}\right)$of $\mathscr{P} / \mathscr{P}^{+} \cong \mathscr{P}$. An intertwining operator $V_{1} \rightarrow V_{2}$ between admissible representations $\left(\rho_{1}, V_{1}\right)$ and $\left(\rho_{2}, V_{2}\right)$ of $G$ defines a canonical $\mathscr{P}$-intertwiner $V_{1}^{\mathscr{P}^{+}} \rightarrow V_{2}^{\mathscr{P}^{+}}$.

Definition 2.1. The parahoric restriction functor for $\mathscr{P}$ is the exact functor between categories of admissible complex linear representations

$$
\mathbf{r}_{\mathscr{P}}: \operatorname{Rep}(G) \rightarrow \operatorname{Rep}(\underline{\mathscr{P}}), \quad\left\{\begin{array}{l}
(\rho, V) \mapsto\left(\rho \mid \mathscr{P}, V^{\mathscr{P}+}\right), \\
\left(V_{1} \rightarrow V_{2}\right) \mapsto\left(V_{1}^{\mathscr{P}^{+}} \rightarrow V_{2}^{\mathscr{P}+}\right) .
\end{array}\right.
$$

For parahoric subgroups $\mathscr{P}_{2} \subseteq \mathscr{P}_{1} \subseteq G$, parahoric restriction is transitive

$$
\mathbf{r}_{\mathscr{P}_{2}}(\rho, V) \cong \mathbf{r}_{\mathscr{P}_{2} / \mathscr{P}_{1}^{+}} \circ \mathbf{r}_{\mathscr{P}_{1}}(\rho, V)
$$

where $\mathbf{r}_{\mathscr{P}_{2} / \mathscr{P}_{1}^{+}}: \operatorname{Rep}\left(\underline{\mathscr{P}_{1}}\right) \rightarrow \operatorname{Rep}\left(\underline{\mathscr{P}_{2}}\right)$ is the parabolic restriction functor with respect to the parabolic subgroup $\mathscr{P}_{2} / \mathscr{P}_{1}^{+} \subseteq \mathscr{P}_{1} / \mathscr{P}_{1}^{+} \cong \underline{\mathscr{P}_{1}}$ and Levi quotient $\underline{\mathscr{P}_{2}}$. Compare Vignéras [13, 4.1.3].

The depth of an irreducible admissible representation $\rho$ of $G$ is defined in the sense of Moy and Prasad [5]. By definition, an irreducible smooth representation of $G$ has depth zero if and only if it admits non-zero parahoric restriction with respect to some parahoric subgroup. 
Let $P$ be a parabolic subgroup of $G$ with Levi subgroup $M$ and let $S \subseteq M$ be a maximal $F$-split torus of $G$. Fix an admissible irreducible representation $\sigma$ of $M$ and an irreducible subquotient $\rho$ of its parabolic induction to $G$. Let $\mathscr{P}$ be a parahoric subgroup attached to a point in the apartment of $S$.

Proposition 2.2. If $\sigma$ has non-zero parahoric restriction with respect to the parahoric subgroup $M \cap \mathscr{P}$ of $M$, then $\rho$ has non-zero parahoric restriction with respect to $\mathscr{P}$.

Proof. Replace $P$ by an associate parabolic with the same Levi subgroup, so that there is a monomorphism $\rho \hookrightarrow \operatorname{Ind}_{P}^{G}(\sigma)$ in $\operatorname{Rep}(G)$ [5, 2.5]. By Frobenius reciprocity, there is an epimorphism $\mathbf{r}_{P}(\rho) \rightarrow \sigma$ in $\operatorname{Rep}(M)$, where $\mathbf{r}_{P}$ denotes Jacquet's functor from $\operatorname{Rep}(G)$ to $\operatorname{Rep}(M)$. By exactness of the functor of $\mathscr{P}^{+} \cap M$-invariants there is an epimorphism

$$
\left(\mathbf{r}_{P}(\rho)\right)^{M \cap \mathscr{P}^{+}} \rightarrow \sigma^{M \cap \mathscr{P}^{+}} .
$$

Since $M \cap \mathscr{P}^{+}$is the pro-unipotent radical of $M \cap \mathscr{P}$, the right hand side is non-zero by assumption and therefore $\left(\mathbf{r}_{P}(\rho)\right)^{M \cap \mathscr{P}^{+}}$is also non-zero. Since $\mathscr{P}^{+} \subseteq G$ admits Iwahori decomposition with respect to $P$ and $M$ [5, 4.2], there is a surjection of vector spaces $\rho^{\mathscr{P}^{+}} \rightarrow\left(\mathbf{r}_{P^{\prime}}(\rho)\right)^{M \cap \mathscr{P}^{+}}$[5, 2.2]. Especially, $\rho^{\mathscr{P}^{+}}$is non-zero.

\subsection{Parahoric restriction for GL(1) and GL(2)}

For $G=\mathrm{GL}(1, F)$, the irreducible admissible representations are the smooth characters $\mu: F^{\times} \rightarrow \mathbb{C}^{\times}$. The unique parahoric subgroup is $\mathfrak{o}^{\times}$with pro-unipotent radical $1+\mathfrak{p}$. If $\mu$ is tamely ramified or unramified, its parahoric restriction $\mathbf{r}_{\mathfrak{o}} \times(\mu)$ is the character $\widetilde{\mu}: \mathfrak{o}^{\times} /(1+\mathfrak{p}) \rightarrow \mathbb{C}$ such that

$$
\mu(x)=\widetilde{\mu}(x(1+\mathfrak{p})) \quad \text { for } \quad x \in \mathfrak{o}^{\times} .
$$

If $\mu$ is wildly ramified, its parahoric restriction is $\widetilde{\mu}=0$.

For $G=\mathrm{GL}(2, F)$ fix the standard Borel $B$ of upper triangular matrices and the maximal torus $T$ of diagonal matrices. The conjugacy classes of parahoric subgroups are represented by the standard hyperspecial parahoric subgroup $\mathscr{K}=\mathscr{K}_{G}=\operatorname{GL}(2, \mathfrak{o})$

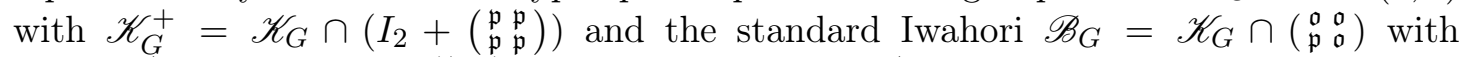
$\mathscr{B}=\mathscr{B}_{G}^{+}=\mathscr{B}_{G} \cap\left(I_{2}+\left(\begin{array}{c}\mathfrak{p} \mathfrak{o} \\ \mathfrak{p} \mathfrak{p}\end{array}\right)\right)$. We identify $\mathscr{K}_{G} / \mathscr{K}_{G}^{+} \cong \mathrm{GL}(2, \mathfrak{o} / \mathfrak{p}) \cong \mathrm{GL}(2, q)$ by the canonical isomorphism and identify $\mathscr{B}_{G} / \mathscr{B}_{G}^{+} \cong(\mathfrak{o} / \mathfrak{p})^{\times} \times(\mathfrak{o} / \mathfrak{p})^{\times}$via $x \mapsto\left(x_{11} \mathfrak{p}, x_{22} \mathfrak{p}\right)$.

The irreducible smooth representations of $G$ are

1. the principal series $\mu_{1} \times \mu_{2}=\operatorname{Ind}_{B}^{G}\left(\mu_{1} \otimes \mu_{2}\right)$ with $\mu_{1} \mu_{2}^{-1} \neq \nu^{ \pm 1}$,

2. one-dimensional representations $\mu_{1} \mathbf{1}_{G}=\mu_{1}$ 。 det,

3. twists of the Steinberg representation $\mu_{1} \mathrm{St}_{G}=\left(\mu_{1} \circ \mathrm{det}\right) \otimes \mathrm{St}_{G}$,

4. cuspidal irreducible representations $\pi$ 
Table 2: Parahoric restriction for smooth irreducible representations of GL $(2, F)$.

\begin{tabular}{lcc}
\hline$\rho$ of $\mathrm{GL}(2, F)$ & $\mathbf{r}_{\mathscr{K}}(\rho)$ of $\mathrm{GL}(2, q)$ & $\mathbf{r}_{\mathscr{B}}(\rho)$ of $\mathrm{GL}(1, q) \times \mathrm{GL}(1, q)$ \\
\hline$\mu_{1} \times \mu_{2}$ & $\widetilde{\mu_{1}} \times \widetilde{\mu_{2}}$ & $\widetilde{\mu_{1}} \nabla \widetilde{\mu_{2}}+\widetilde{\mu_{2}} \otimes \widetilde{\mu_{1}}$ \\
$\mu_{1} \cdot \mathbf{1}_{\mathrm{GL}(2, F)}$ & $\widetilde{\mu_{1}} \cdot \mathbf{1}_{\mathrm{GL}(2, q)}$ & $\widetilde{\mu_{1}} \otimes \widetilde{\mu_{1}}$ \\
$\mu_{1} \cdot \mathrm{St}_{\mathrm{GL}(2, F)}$ & $\widetilde{\mu_{1}} \cdot \mathrm{St}_{\mathrm{GL}(2, q)}$ & 0 \\
$\pi$ depth zero & cuspidal irreducible & 0 \\
$\pi$ positive depth & 0 & 0 \\
\hline
\end{tabular}

for smooth characters $\mu_{1}, \mu_{2}$ of $F^{\times}$.

Lemma 2.3. The parahoric restriction of the irreducible admissible representations $\rho$ of $G$ at $\mathscr{K}$ and $\mathscr{B}$ is given by Table $23^{3}$

Proof. For a pair of smooth characters $\mu_{1}, \mu_{2}$ of $F^{\times}$, the parahoric restriction of $\mu_{1} \times \mu_{2}$ at $\mathscr{K}_{G}$ is

$$
\mathbf{r}_{\mathscr{K}_{G}}\left(\mu_{1} \times \mu_{2}\right) \cong \widetilde{\mu_{1}} \times \widetilde{\mu_{2}},
$$

by a standard argument using Iwasawa decomposition $G=B \mathscr{K}_{G}$, compare Prop. 3.3 By exactness of parahoric restriction and Maschke's theorem, the exact sequence

$$
0 \longrightarrow \mu_{1} \mathbf{1}_{\mathrm{GL}(2, F)} \longrightarrow \nu^{-1 / 2} \mu_{1} \times \nu^{1 / 2} \mu_{1} \longrightarrow \mu_{1} \mathrm{St}_{\mathrm{GL}(2, F)} \longrightarrow 0 .
$$

yields an isomorphism

$$
\widetilde{\mu_{1}} \operatorname{St}_{\mathrm{GL}(2, q)} \oplus \widetilde{\mu_{1}} \mathbf{1}_{\mathrm{GL}(2, q)} \cong \widetilde{\mu_{1}} \times \widetilde{\mu_{1}} \cong \mathbf{r}_{\mathscr{K}_{G}}\left(\mu_{1} \mathbf{1}_{G}\right) \oplus \mathbf{r}_{\mathscr{K}_{G}}\left(\mu_{1} \mathrm{St}_{G}\right)
$$

If $\mu_{1}$ is tamely ramified or unramified, we have $\mathbf{r}_{\mathscr{K}_{G}}\left(\mu_{1} \circ\right.$ det $)=\widetilde{\mu_{1}} \circ$ det because $\operatorname{det}\left(\mathscr{K}_{G}^{+}\right)=1+\mathfrak{p}$. Cuspidal irreducible representations of depth zero are compactly induced from the normalizer of $\mathscr{K}$ [5, 6.8], the result is then implied by a theorem of Vignéras [12, Cor. 5.3]. For Iwahori restriction use transitivity (3).

\subsection{The group $\operatorname{GSp}(4)$}

The group $\mathbf{G}=\mathrm{GSp}(4)$ of symplectic similitudes of genus two is defined over $\mathbb{Z}$ by the equation

$$
J=\nu g J g^{t} \quad \text { for } \quad g \in \mathrm{GL}(4), \nu \in \mathrm{GL}(1) \quad \text { and } \quad J=\left({ }_{-I_{2}} I_{2}\right) .
$$

The similitude factor $\operatorname{sim}(g)=\nu$ is uniquely determined by $g$ and defines a character sim : $\mathbf{G} \rightarrow \mathrm{GL}(1)$. We fix the split torus $\mathbf{T}$ of diagonal matrices and the standard parabolic subgroups

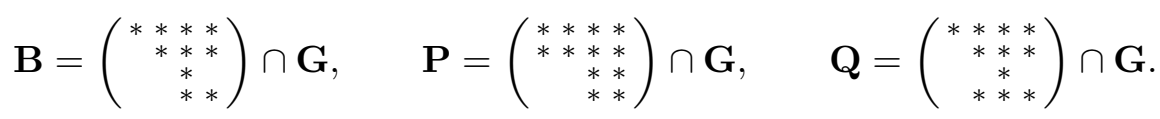

\footnotetext{
${ }^{3}$ This is well-known, compare Vignéras [11, III.3.14] or Bushnell and Henniart [3, $\left.\S 14, \S 15\right]$.
} 
The corresponding groups of $F$-rational points are $G, T, B, P, Q$.

For smooth characters $\mu_{i}$ of $F^{\times}, i=0,1,2$, normalized parabolic induction of the character $T \rightarrow \mathbb{C}, \operatorname{diag}\left(t_{1}, t_{2}, t_{0} / t_{1}, t_{0} / t_{2}\right) \mapsto \mu_{1}\left(t_{1}\right) \mu_{2}\left(t_{2}\right) \mu_{0}\left(t_{0}\right)$ via the standard Borel $B$ yields the admissible representation $\mu_{1} \times \mu_{2} \rtimes \mu_{0}$ of $G$. For parabolic induction via $P$ and $Q$ the notation is analogous, compare Tadić [10].

A non-trivial additive character $\psi: F \rightarrow \mathbb{C}^{\times}$gives rise to a generic character of $U_{B}$ via $\psi_{U}: U_{B} \rightarrow \mathbb{C}, u \mapsto \psi\left(u_{12}+u_{24}\right)$. An admissible representation $\rho$ of $G$ is generic if it admits a non-trivial $U_{B}$-intertwining operator $\left(\left.\rho\right|_{U_{B}}, V\right) \rightarrow\left(\psi_{U}, \mathbb{C}\right)$.

We review the classification of standard parahoric subgroups of $\operatorname{GSp}(4, F)$. The character group $X^{*}(\mathbf{T})=\operatorname{Hom}(\mathbf{T}, \mathrm{GL}(1))$ is generated as a free group by the characters $e_{i}$ : $\operatorname{diag}\left(t_{1}, t_{2}, t_{0} / t_{1}, t_{0} / t_{2}\right) \mapsto t_{i}$ for $i=0,1,2$. The simple affine roots $\psi_{0}=-\left(2 e_{1}-e_{0}\right)+$ $1, \psi_{1}=e_{1}-e_{2}$ and $\psi_{2}=2 e_{2}-e_{0}$ constitute the affine Dynkin diagram

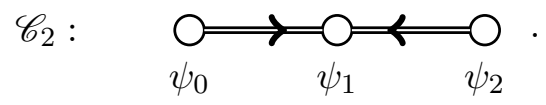

Let $N(T)$ be the normalizer of $T$ in $G$. The affine Weyl group $N(T) / \mathbf{T}(\mathfrak{o})$ is generated by the root reflections $s_{i}$ at $\psi_{i}$ for $i=0,1,2$ and the Atkin-Lehner element $u_{1}$

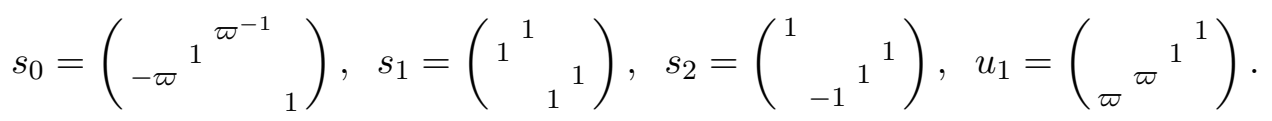

The simple affine roots $\psi_{0}$ and $\psi_{2}$ are conjugate under $u_{1}$. The closed standard alcove $\mathcal{C}$ in the apartment attached to $T$ is defined by $\psi_{i}(x) \geq 0$ for $i=0,1,2$. To each facet in $\mathcal{C}$ is attached one of the standard parahoric subgroups of $\operatorname{GSp}(4, F)$ :

1. the standard Iwahori subgroup $\mathscr{B}$, attached to $\mathcal{C}$,

$$
\mathscr{B}=\operatorname{sim}^{-1}\left(\mathfrak{o}^{\times}\right) \cap\left(\begin{array}{cccc}
\mathfrak{o} & \mathfrak{o} & \mathfrak{o} & \mathfrak{o} \\
\mathfrak{p} & \mathfrak{o} & \mathfrak{o} & \mathfrak{o} \\
\mathfrak{p} & \mathfrak{p} & \mathfrak{o} & \mathfrak{p} \\
\mathfrak{p} & \mathfrak{p} & \mathfrak{o} & \mathfrak{o}
\end{array}\right), \quad \mathscr{B}^{+}=\mathscr{B} \cap\left(\begin{array}{cccc}
1+\mathfrak{p} & \mathfrak{o} & \mathfrak{o} & \mathfrak{o} \\
\mathfrak{p} & 1+\mathfrak{p} & \mathfrak{o} & \mathfrak{o} \\
\mathfrak{p} & \mathfrak{p} & 1+\mathfrak{p} & \mathfrak{p} \\
\mathfrak{p} & \mathfrak{p} & \mathfrak{o} & 1+\mathfrak{p}
\end{array}\right)
$$

with Levi quotient $\mathscr{B} / \mathscr{B}^{+} \cong \mathrm{GL}(1, q)^{3}$ via $x \mapsto\left(x_{11}, x_{22}, \operatorname{sim}(x)\right)$,

2. the standard Siegel parahoric $\mathscr{P}$, attached to the facet $\psi_{1}^{-1}(0) \cap \mathcal{C}$,

$$
\mathscr{P}=\operatorname{sim}^{-1}\left(\mathfrak{o}^{\times}\right) \cap\left(\begin{array}{cccc}
\mathfrak{o} & \mathfrak{o} & \mathfrak{o} & \mathfrak{o} \\
\mathfrak{o} & \mathfrak{o} & \mathfrak{o} & \mathfrak{o} \\
\mathfrak{p} & \mathfrak{p} & \mathfrak{o} & \mathfrak{o} \\
\mathfrak{p} & \mathfrak{p} & \mathfrak{o} & \mathfrak{o}
\end{array}\right), \quad \mathscr{P}^{+}=\mathscr{P} \cap\left(\begin{array}{cccc}
1+\mathfrak{p} & \mathfrak{p} & \mathfrak{o} & \mathfrak{o} \\
\mathfrak{p} & 1+\mathfrak{p} & \mathfrak{o} & \mathfrak{o} \\
\mathfrak{p} & \mathfrak{p} & 1+\mathfrak{p} & \mathfrak{p} \\
\mathfrak{p} & \mathfrak{p} & \mathfrak{p} & 1+\mathfrak{p}
\end{array}\right)
$$

with $\mathscr{P} / \mathscr{P}^{+} \cong \mathrm{GL}(2, q) \times \mathrm{GL}(1, q)$ via $x \mapsto\left(\left(\begin{array}{cc}x_{11} & x_{12} \\ x_{21} & x_{22}\end{array}\right), \operatorname{sim}(x)\right)$, 
3. the standard Klingen parahoric $\mathscr{Q}$, attached to the facet $\psi_{2}^{-1}(0) \cap \mathcal{C}$,

$$
\mathscr{Q}=\operatorname{sim}^{-1}\left(\mathfrak{o}^{\times}\right) \cap\left(\begin{array}{cccc}
\mathfrak{o} & \mathfrak{o} & \mathfrak{o} & \mathfrak{o} \\
\mathfrak{p} & \mathfrak{o} & \mathfrak{o} & \mathfrak{o} \\
\mathfrak{p} & \mathfrak{p} & \mathfrak{o} & \mathfrak{p} \\
\mathfrak{p} & \mathfrak{o} & \mathfrak{o} & \mathfrak{o}
\end{array}\right), \quad \mathscr{Q}^{+}=\mathscr{Q} \cap\left(\begin{array}{cccc}
1+\mathfrak{p} & \mathfrak{o} & \mathfrak{o} & \mathfrak{o} \\
\mathfrak{p} & 1+\mathfrak{p} & \mathfrak{o} & \mathfrak{p} \\
\mathfrak{p} & \mathfrak{p} & 1+\mathfrak{p} & \mathfrak{p} \\
\mathfrak{p} & \mathfrak{p} & \mathfrak{o} & 1+\mathfrak{p}
\end{array}\right)
$$

with $\mathscr{Q} / \mathscr{Q}^{+} \cong \mathrm{GL}(1, q) \times \mathrm{GSp}(2, q)$ via $x \mapsto\left(x_{11},\left(\begin{array}{ccc}x_{22} & x_{24} \\ x_{42} & x_{44}\end{array}\right)\right)$,

4. the standard hyperspecial parahoric subgroup $\mathscr{K}=\operatorname{GSp}(4, \mathfrak{o})$, attached to the facet $\psi_{1}^{-1}(0) \cap \psi_{2}^{-1}(0) \cap \mathcal{C}$,

$$
\mathscr{K}=\operatorname{sim}^{-1}\left(\mathfrak{o}^{\times}\right) \cap\left(\begin{array}{cccc}
\mathfrak{o} & \mathfrak{o} & \mathfrak{o} & \mathfrak{o} \\
\mathfrak{o} & \mathfrak{o} & \mathfrak{o} & \mathfrak{o} \\
\mathfrak{o} & \mathfrak{o} & \mathfrak{o} & \mathfrak{o} \\
\mathfrak{o} & \mathfrak{o} & \mathfrak{o} & \mathfrak{o}
\end{array}\right), \quad \mathscr{K}^{+}=\mathscr{K} \cap\left(\begin{array}{cccc}
1+\mathfrak{p} & \mathfrak{p} & \mathfrak{p} & \mathfrak{p} \\
\mathfrak{p} & 1+\mathfrak{p} & \mathfrak{p} & \mathfrak{p} \\
\mathfrak{p} & \mathfrak{p} & 1+\mathfrak{p} & \mathfrak{p} \\
\mathfrak{p} & \mathfrak{p} & \mathfrak{p} & 1+\mathfrak{p}
\end{array}\right)
$$

with the canonical map $\mathscr{K} / \mathscr{K}^{+} \cong \mathrm{GSp}(4, \mathfrak{o} / \mathfrak{p}) \cong \mathrm{GSp}(4, q)$,

5. the standard paramodular subgroup $\mathscr{J}$ with facet $\psi_{0}^{-1}(0) \cap \psi_{2}^{-1}(0) \cap \mathcal{C}$,

$$
\mathscr{J}=\operatorname{sim}^{-1}\left(\mathfrak{o}^{\times}\right) \cap\left(\begin{array}{cccc}
\mathfrak{o} & \mathfrak{o} & \mathfrak{p}^{-1} & \mathfrak{o} \\
\mathfrak{p} & \mathfrak{o} & \mathfrak{o} & \mathfrak{o} \\
\mathfrak{p} & \mathfrak{p} & \mathfrak{o} & \mathfrak{p} \\
\mathfrak{p} & \mathfrak{o} & \mathfrak{o} & \mathfrak{o}
\end{array}\right), \quad \mathscr{J}^{+}=\mathscr{J} \cap\left(\begin{array}{cccc}
1+\mathfrak{p} & \mathfrak{o} & \mathfrak{o} & \mathfrak{o} \\
\mathfrak{p} & 1+\mathfrak{p} & \mathfrak{o} & \mathfrak{p} \\
\mathfrak{p}^{2} & \mathfrak{p} & 1+\mathfrak{p} & \mathfrak{p} \\
\mathfrak{p} & \mathfrak{p} & \mathfrak{o} & 1+\mathfrak{p}
\end{array}\right)
$$

with $\mathscr{J} / \mathscr{J}^{+} \cong\left(\mathrm{GL}(2, q)^{2}\right)^{0}:=\left\{(a, b) \in \mathrm{GL}(2, q)^{2} \mid \operatorname{det} a=\operatorname{det} b\right\}$ via

$$
x \mapsto\left(\left(\begin{array}{cc}
x_{11} & x_{13} \varpi \\
x_{31} \varpi^{-1} & x_{33}
\end{array}\right),\left(\begin{array}{ll}
x_{22} & x_{24} \\
x_{42} & x_{44}
\end{array}\right)\right),
$$

6. the parahoric $u_{1}^{-1} \mathscr{Q} u_{1}$ attached to the facet $\psi_{0}^{-1}(0) \cap \mathcal{C}$,

7. the hyperspecial parahoric $u_{1}^{-1} \mathscr{K} u_{1}$ attached to $\psi_{0}^{-1}(0) \cap \psi_{1}^{-1}(0) \cap \mathcal{C}$.

Conjugation by the Atkin-Lehner element $u_{1}$ preserves $\mathscr{B}, \mathscr{P}$ and $\mathscr{J}$. The standard maximal parahorics are $\mathscr{K}, \mathscr{J}$ and $u_{1}^{-1} \mathscr{K} u_{1}$.

There are double coset decompositions

$$
\operatorname{GSp}(4, F)=B \mathscr{K}=P \mathscr{J}=Q \mathscr{J} \sqcup Q s_{1} \mathscr{J}
$$

for the standard parabolics $B, P, Q$, where $\sqcup$ denotes the disjoint union. The proof is elementary and follows from Iwasawa and Bruhat decomposition.

For every parahoric subgroup of $\operatorname{GSp}(4, F)$, the image of the pro-unipotent radical under the similitude character is $1+\mathfrak{p}$. Therefore twisting a representation $\rho$ by a tamely ramified or unramified character $\mu$ of $F^{\times}$commutes with parahoric restriction in the following sense:

$$
\begin{aligned}
& \mathbf{r}_{\mathscr{K}}((\mu \circ \operatorname{sim}) \otimes \rho) \cong(\widetilde{\mu} \circ \operatorname{sim}) \otimes \mathbf{r}_{\mathscr{K}}(\rho), \\
& \mathbf{r}_{\mathscr{J}}((\mu \circ \operatorname{sim}) \otimes \rho) \cong(\widetilde{\mu} \circ \operatorname{det}) \otimes \mathbf{r}_{\mathscr{J}}(\rho) .
\end{aligned}
$$

with det : $\left(\operatorname{GL}(2, q)^{2}\right)^{0} \rightarrow \mathbb{F}_{q}^{\times},(a, b) \mapsto \operatorname{det} a$. 


\section{Proof of Theorem 1.1}

Proposition 3.1. For an admissible representation $\left(\sigma, V_{\sigma}\right)$ of $\operatorname{GSp}(2, F)$ and a character $\mu_{1}: F^{\times} \rightarrow \mathbb{C}^{\times}$, the parahoric restriction at $\mathscr{J}$ of the Klingen induced representation $\mu_{1} \rtimes \sigma$ is

$$
\mathbf{r}_{\mathscr{J}}\left(\mu_{1} \rtimes \sigma\right) \cong\left[\widetilde{\mu_{1}} \times 1, \widetilde{\sigma}\right] \oplus\left[\widetilde{\sigma}, \widetilde{\mu_{1}} \times 1\right]
$$

for $\widetilde{\mu_{1}}=\mathbf{r}_{\mathfrak{o}} \times\left(\mu_{1}\right)$ and $\widetilde{\sigma}=\mathbf{r}_{\mathrm{GL}(2, \mathfrak{o})}(\sigma)$.

Proof. An explicit model of $\mathbf{r}_{\mathscr{J}}\left(\mu_{1} \rtimes \sigma\right)$ is given by the right-action of $\mathscr{J}$ on

$$
\widetilde{V}=\left\{f: G \rightarrow V_{\sigma} \mid f(p g k)=\delta_{Q}^{1 / 2}(p)\left(\mu_{1} \otimes \sigma\right)(p) f(g) \forall p \in Q, g \in G, k \in \mathscr{J}^{+}\right\} .
$$

By (5), any $f \in \widetilde{V}$ is uniquely determined by its restriction to $\mathscr{J}$ and $s_{1} \mathscr{J}$, so the $\mathscr{J}$-representation $\widetilde{V}$ is isomorphic to the direct sum

$$
\left\{\left.f\right|_{\mathscr{J}}: \mathscr{J} \rightarrow V_{\sigma} \mid f \in \widetilde{V}\right\} \oplus\left\{\left.f\right|_{s_{1} \mathscr{J}}: s_{1} \mathscr{J} \rightarrow V_{\sigma} \mid f \in \widetilde{V}\right\} .
$$

Every $\left.f\right|_{\mathscr{J}}$ in the first subspace is left invariant under $\mathscr{J}^{+} \cap Q$, so it maps to the $\sigma$ invariants under $\operatorname{GSp}(2, \mathfrak{o})^{+}$. Thus $\left.f\right|_{\mathscr{J}}$ factors over a unique function

$$
\widetilde{f}: \mathscr{J} / \mathscr{J}^{+} \rightarrow V_{\sigma}^{\mathrm{GSp}(2, \mathfrak{o})^{+}} \quad \text { with } \quad \widetilde{f}(q g)=\widetilde{\mu}\left(q_{11}\right) \widetilde{\sigma}\left(\begin{array}{cc}
q_{22} & q_{24} \\
q_{42} & q_{44}
\end{array}\right) \widetilde{f}(g)
$$

for every $g \in \mathscr{J} / \mathscr{J}^{+} \cong\left(\mathrm{GL}(2, q)^{2}\right)^{0}$ and every

$$
q \in(\mathscr{J} \cap Q) \mathscr{J}^{+} / \mathscr{J}^{+} \cong\left(\left(\begin{array}{c}
* * \\
*
\end{array}\right) \times\left(\begin{array}{c}
* * \\
* *
\end{array}\right)\right) \cap\left(\mathrm{GL}(2, q)^{2}\right)^{0} .
$$

By definition of the isomorphism $\mathscr{J} / \mathscr{J}^{+} \cong\left(\operatorname{GL}(2, q)^{2}\right)^{0}$, the space of these $\tilde{f}$ is the induced representation $[\widetilde{\mu} \times 1, \widetilde{\sigma}]$.

For the second subspace the argument is analogous.

Proposition 3.2. For smooth characters $\mu_{0}, \mu_{1}, \mu_{2}$ of $F^{\times}$and an irreducible admissible representation $\left(\sigma, V_{\sigma}\right)$ of $\mathrm{GL}(2, F)$, the parahoric restriction of the Siegel induced representation $\sigma \rtimes \mu_{0}$ at the standard paramodular subgroup $\mathscr{J}$ is

$$
\mathbf{r}_{\mathscr{J}}\left(\sigma \rtimes \mu_{0}\right) \cong \begin{cases}\widetilde{\mu_{0}}\left[1 \times \widetilde{\mu_{1}}, 1 \times \widetilde{\mu_{2}}\right]+\widetilde{\mu_{0}}\left[1 \times \widetilde{\mu_{2}}, 1 \times \widetilde{\mu_{1}}\right] & \sigma \cong \mu_{1} \times \mu_{2}, \\ \widetilde{\mu_{0}}\left[1 \times \widetilde{\mu_{1}}, 1 \times \widetilde{\mu_{1}}\right] & \sigma=\mu_{1} \mathrm{St}, \mu_{1} \mathbf{1}, \\ 0 & \sigma \text { cuspidal. }\end{cases}
$$

Proof. By (7) we can assume without loss of generality that $\mu_{0}=1$. An explicit model $\widetilde{V}$ of $\mathbf{r}_{\mathscr{J}}(\sigma \rtimes 1)$ is given by right-multiplication with elements of $\mathscr{J}$ on the vector space of smooth functions $f: G \rightarrow V_{\sigma}$ with

$$
f(p g k)=\delta_{P}^{1 / 2}(p) \cdot \sigma\left(\begin{array}{ll}
p_{11} & p_{12} \\
p_{21} & p_{22}
\end{array}\right) f(g)
$$


for $p \in P, g \in G$ and $k \in \mathscr{J}^{+}$. By the decomposition $G=P \mathscr{J}$ (5), every such $f$ is uniquely determined by its restriction to $\mathscr{J}$. Therefore $\widetilde{V}$ is isomorphic to the vector space of $\mathscr{J}^{+}$-invariant functions

$$
\widetilde{f}: \mathscr{J} \rightarrow V_{\sigma}
$$

which satisfy the condition

$$
\widetilde{f}(p g)=\sigma\left(\begin{array}{ll}
p_{11} & p_{12} \\
p_{21} & p_{22}
\end{array}\right) \widetilde{f}(g) \quad \forall g \in \mathscr{J}, \quad \forall p \in P \cap \mathscr{J} .
$$

Since $\tilde{f}$ is left-invariant under every $p \in P \cap \mathscr{J}^{+}$, the value $\widetilde{f}(g) \in V_{\sigma}$ is invariant under $\left(\begin{array}{cc}1+\mathfrak{p} & \mathfrak{o} \\ \mathfrak{p} & 1+\mathfrak{p}\end{array}\right) \subseteq \mathrm{GL}(2, F)$. That means $\widetilde{f}(g)$ must be contained in the parahoric restriction $\mathbf{r}_{\mathscr{B}_{\mathrm{GL}(2)}}(\sigma)$ with respect to the standard Iwahori $\mathscr{B}_{\mathrm{GL}(2)} \subseteq \mathrm{GL}(2, F)$. By Lemma 2.3 . $\mathbf{r}_{\mathscr{B}_{\mathrm{GL}(2)}}(\sigma)$ is zero for cuspidal $\sigma$. For $\sigma=\mu \mathbf{1}, \mu \mathrm{St}$ it is isomorphic to $\widetilde{\mu_{1}} \nabla \widetilde{\mu_{1}}$ and the condition on $\widetilde{f}$ is

$$
\widetilde{f}(p g)=\widetilde{\mu_{1}}\left(p_{11}\right) \widetilde{\mu_{1}}\left(p_{22}\right) \widetilde{f}(g), \quad \forall g \in \mathscr{J} / \mathscr{J}^{+}, \quad \forall p \in(P \cap \mathscr{J}) /\left(P \cap \mathscr{J}^{+}\right) .
$$

By construction of the isomorphism $\mathscr{J} / \mathscr{J}^{+} \cong\left(\mathrm{GL}(2, q)^{2}\right)^{0}$, the action of $\mathscr{J}$ on $\widetilde{V}$ is the induced representation $\left[1 \times \widetilde{\mu_{1}}, 1 \times \widetilde{\mu_{1}}\right]$. For the principal series $\sigma=\mu_{1} \times \mu_{2}$, the argument is analogous.

Proposition 3.3. For admissible representations of $\mathrm{GSp}(4, F)$ that are parabolically induced, the parahoric restriction at $\mathscr{K}$ is given by

$$
\begin{gathered}
\mathbf{r}_{\mathscr{K}}\left(\mu_{1} \times \mu_{2} \rtimes \mu_{0}\right) \cong \widetilde{\mu_{1}} \times \widetilde{\mu_{2}} \rtimes \widetilde{\mu_{0}}, \\
\mathbf{r}_{\mathscr{K}}\left(\mu_{1} \rtimes \sigma\right) \cong \widetilde{\mu_{1}} \rtimes \mathbf{r}_{\mathrm{GSp}(2, \mathfrak{o})}(\sigma), \\
\mathbf{r}_{\mathscr{K}}\left(\sigma \rtimes \mu_{0}\right) \cong \mathbf{r}_{\mathrm{GL}(2, \mathfrak{o})}(\sigma) \rtimes \widetilde{\mu_{0}},
\end{gathered}
$$

for smooth characters $\mu_{i}: F^{\times} \rightarrow \mathbb{C}^{\times}$and an admissible representation $\sigma$ of $\mathrm{GL}(2, F)$.

Proof. The proof is similar to the previous propositions using $G=B \mathscr{K}$.

Thm.1.1 i). We only discuss the case of odd $q$; for even $q$ the proof is analogous. Irreducible representations $\rho$ of type I, II, III, VII and X are parabolically induced, so the result is clear by Prop. 3.3 , by (4) and Table 2. Otherwise, $\rho$ is a non-trivial subquotient of a parabolically induced representation $\kappa$ and $\mathbf{r}_{\mathscr{K}}(\kappa)$ is given by Prop. 3.3. If $\mathbf{r}_{\mathscr{K}}(\kappa)=0$, then $\mathbf{r}_{\mathscr{K}}(\rho)=0$ by exactness, otherwise $\mathbf{r}_{\mathscr{K}}(\rho)$ is a non-zero subquotient of $\mathbf{r}_{\mathscr{K}}(\kappa)$ by Prop. 2.2. It remains to determine the correct constituents of $\mathbf{r}_{\mathscr{K}}(\kappa)$ case by case. By (6) we can assume without loss of generality that $\mu_{0}=1$.

For the trivial representation $\rho=\mathbf{1}_{\mathrm{GSp}(4, F)}$ (type IVd), the hyperspecial parahoric restriction is trivial $\mathbf{r}_{\mathscr{K}}(\rho)=\theta_{0}$. By [6, (2.9)] and character theory [9]

$$
\mathbf{r}_{\mathscr{K}}\left(L\left(\nu^{2}, \nu^{-1} \mathrm{St}_{\mathrm{GSp}(2, F)}\right)\right)+\mathbf{r}_{\mathscr{K}}\left(\mathbf{1}_{\mathrm{GSp}(4, F)}\right) \cong \mathbf{1}_{\mathrm{GL}(2, q)} \rtimes 1=\chi_{3}(1,1)
$$


decomposes as $\chi_{3}(1,1)=\theta_{0}+\theta_{1}+\theta_{3}$, so for type IVb

$$
\mathbf{r}_{\mathscr{K}}\left(L\left(\nu^{2}, \nu^{-1} \mathrm{St}_{\mathrm{GSp}(2, F)}\right)\right)=\theta_{1}+\theta_{3} .
$$

By the same argument we determine the parahoric restriction for type IVa and IVc as constituents of $\chi_{1}(1,1)=1 \rtimes \mathbf{1}_{\mathrm{GSp}(2, q)}$ and $\chi_{2}(1,1)=1 \rtimes \mathrm{St}_{\mathrm{GSp}(2, q)}$.

The representation $\rho=L\left(\nu^{1 / 2} \xi \mathrm{St}, \nu^{-1 / 2}\right)$ of type $\mathrm{Vb}$ is a constituent of both

$$
\nu^{1 / 2} \xi \mathrm{St}_{\mathrm{GL}(2, F)} \rtimes \nu^{-1 / 2} \text { and } \nu^{1 / 2} \xi \mathbf{1}_{\mathrm{GL}(2, F)} \rtimes \nu^{-1 / 2} \xi
$$

[6, (2.10)]. Therefore the parahoric restriction $\mathbf{r}_{\mathscr{K}}(\rho)$ must be a (non-zero) constituent of both $\widetilde{\xi} \operatorname{St}_{\mathrm{GL}(2, q)} \rtimes 1$ and $\widetilde{\xi} \mathrm{St}_{\mathrm{GL}(2, q)} \rtimes \widetilde{\xi}$. By [9], the only common constituent is $\theta_{1}$ for unramified $\xi$ and $\tau_{2}$ for tamely ramified $\xi$. By exactness, types Va, Vc and Vd are clear.

The Klingen induced representation $1 \rtimes \mathrm{St}_{\mathrm{GL}(2, F)}$ splits into the direct sum of $\tau\left(S, \nu^{-1 / 2}\right)$ of type VIa and $\tau\left(T, \nu^{-1 / 2}\right)$ of type VIb [6, (2.11)]. Its parahoric restriction is $\mathbf{r}_{\mathscr{K}}(1 \rtimes$ $\left.\mathrm{St}_{\mathrm{GL}(2, F)}\right)=\theta_{1}+\theta_{3}+\theta_{5}$. The representation $\tau\left(S, \nu^{-1 / 2}\right)$ is contained in $\nu^{1 / 2} \mathrm{St} \rtimes \nu^{-1 / 2}$ with restriction at $\mathscr{K}$ given by $\mathrm{St}_{\mathrm{GL}(2, q)} \rtimes 1=\theta_{1}+\theta_{4}+\theta_{5}$; while $\tau\left(T, \nu^{-1 / 2}\right)$ is contained in $\nu^{1 / 2} \mathbf{1} \rtimes \nu^{-1 / 2}$ with restriction $\theta_{0}+\theta_{1}+\theta_{3}$. Therefore the pair of parahoric restrictions $\left(\mathbf{r}_{\mathscr{K}}\left(\tau\left(S, \nu^{-1 / 2}\right)\right), \mathbf{r}_{\mathscr{K}}\left(\tau\left(T, \nu^{-1 / 2}\right)\right)\right)$ is either $\left(\theta_{5}+\theta_{1}, \theta_{3}\right)$ or $\left(\theta_{5}, \theta_{1}+\theta_{3}\right)$. But the virtual representation

$$
\tau\left(S, \nu^{-1 / 2}\right)-\tau\left(T, \nu^{-1 / 2}\right)
$$

is the endoscopic lift of $\left(\mathrm{St}_{\mathrm{GL}(2, F)}, \mathrm{St}_{\mathrm{GL}(2, F)}\right)$ in the sense of [14], so the trace of its parahoric restriction at $\mathscr{K}$ is zero on the $\operatorname{GSp}(4, q)$-conjugacy class stably conjugate to $\operatorname{diag}\left(a^{q}, a, a^{q^{3}}, a^{q^{2}}\right)$ [7, Cor. 4.25] for $a \in \mathbb{F}_{q^{4}}^{\times}$with $a^{q^{2}+1} \in \mathbb{F}_{q}^{\times}$and $a^{q-1} \neq \pm 1$. This implies $\mathbf{r}_{\mathscr{K}}\left(\tau\left(S, \nu^{-1 / 2}\right)\right)=\theta_{5}+\theta_{1}$ and $\mathbf{r}_{\mathscr{K}}\left(\tau\left(T, \nu^{-1 / 2}\right)\right)=\theta_{3}$. Types VIc and VId are clear by exactness.

For an irreducible cuspidal admissible representation $\pi$ of $\mathrm{GL}(2, F)$ of depth zero, the Klingen induced representation $1 \rtimes \pi$ is a direct sum of two irreducible constituents, the generic $\tau(S, \pi)$ of type VIIIa and the non-generic $\tau(T, \pi)$ of type VIIIb. The parahoric restriction $\mathbf{r}_{\mathscr{K}}(1 \rtimes \pi) \cong 1 \rtimes \widetilde{\pi}=X_{3}(\Lambda, 1)=\chi_{7}(\Lambda)+\chi_{8}(\Lambda)$ has two irreducible constituents, so Prop. 2.2 implies that $\mathbf{r}_{\mathscr{K}}(\tau(S, \pi))$ is isomorphic to one of them and $\mathbf{r}_{\mathscr{K}}(\tau(T, \pi))$ is isomorphic to the other. By a suitable character twist (6) we can assume that $\pi$ is unitary. Then the virtual representation $\tau(S, \pi)-\tau(T, \pi)$ is the local endoscopic character lift of the representation $(\pi, \pi)$ of $\mathrm{GL}(2, F)^{2} / \mathrm{GL}(1, F)$ (antidiagonally embedded), compare [14, Thm. 4.5]. For $\alpha, \beta \in \mathbb{F}_{q^{2}}^{\times}$with $\alpha, \beta, \alpha \beta, \alpha \beta^{q} \notin \mathbb{F}_{q}^{\times}$, the trace of $\mathbf{r}_{\mathscr{K}}(\tau(S, \pi)-\tau(T, \pi))$ on the stable conjugacy class with eigenvalues $\alpha \beta, \alpha \beta^{q}, \alpha^{q} \beta, \alpha^{q} \beta^{q}$ is

$$
2\left(\Lambda(\alpha)+\Lambda\left(\alpha^{q}\right)\right)\left(\Lambda(\beta)+\Lambda\left(\beta^{q}\right)\right),
$$

see [7, Cor. 4.20]. By Shinoda's character table [9], this coincides with the character value of $\chi_{8}(\Lambda)-\chi_{7}(\Lambda)$, but not with $\chi_{7}(\Lambda)-\chi_{8}(\Lambda)$. This implies $\mathbf{r}_{\mathscr{K}}(\tau(S, \pi)) \cong \chi_{8}(\Lambda)$ and $\mathbf{r}_{\mathscr{K}}(\tau(T, \pi)) \cong \chi_{7}(\Lambda)$ 
For type IX, see [7, §3.2.1].

Let $\rho=\delta\left(\nu^{1 / 2} \pi, \nu^{-1 / 2}\right)$ be an irreducible representation of type XIa where $\pi$ is a cuspidal irreducible admissible representation of $\operatorname{GL}(2, F)$ with trivial central character. Then $\mathbf{r}_{\mathscr{K}}(\rho)$ must be one of the two irreducible subquotients of

$$
\mathbf{r}_{\mathscr{K}}\left(\nu^{1 / 2} \pi \rtimes \nu^{-1 / 2}\right)=\widetilde{\pi} \rtimes 1=X_{2}(\Lambda, 1)=\chi_{5}\left(\omega_{\Lambda}, 1\right)+\chi_{6}\left(\omega_{\Lambda}, 1\right) .
$$

By [6, Table A.12], $\rho$ has paramodular level $\geq 3$ and therefore does not admit non-zero invariants under the second paramodular congruence subgroup, which is conjugate to

$$
\left(\begin{array}{cccc}
\mathfrak{o} & \mathfrak{p} & \mathfrak{o} & \mathfrak{p} \\
\mathfrak{p} & \mathfrak{o} & \mathfrak{p} & \mathfrak{o} \\
\mathfrak{o} & \mathfrak{p} & \mathfrak{o} & \mathfrak{p} \\
\mathfrak{p} & \mathfrak{o} & \mathfrak{p} & \mathfrak{o}
\end{array}\right) \cap \mathscr{K}
$$

By character theory, $\chi_{5}\left(\omega_{\Lambda}, 1\right)$ admits non-zero invariants under the image of this group in $\mathscr{K} / \mathscr{K}^{+}$, so $\mathbf{r}_{\mathscr{K}}(\rho)$ cannot be $\chi_{5}\left(\omega_{\Lambda}, 1\right)$. The rest follows from exactness and Prop. 2.2

Thm.1.1 ii). By transitivity of parahoric restriction (3), this is implied by Thm. 1.1 . Parabolic restriction for $\operatorname{GSp}(4, q)$ can be determined explicitly by character theory.

For the paramodular subgroup $\mathscr{J} \subseteq \mathrm{GSp}(4, F)$, the Atkin Lehner involution provides a symmetry condition:

Lemma 3.4. Let $\rho$ be an irreducible admissible representation of $\operatorname{GSp}(4, F)$. The parahoric restriction $\mathbf{r}_{\mathscr{J}}(\rho)$ is isomorphic to $(a, b) \mapsto \mathbf{r}_{\mathscr{J}}(\rho)(b, a)$.

Proof. Conjugation by $u_{1}$ preserves $\mathscr{J}$ and $\mathscr{J}^{+}$and gives rise to the automorphism $(a, b) \mapsto(b, a)$ of $\left(\mathrm{GL}(2, q)^{2}\right)^{0} \cong \mathscr{J} / \mathscr{J}^{+}$.

Thm.1.1 iii). By (7), we can assume without loss of generality that $\mu_{0}=1$. The irreducible admissible representations $\rho$ of type I, IIIa, IIIb and VII are Klingen induced and the statement is implied by Prop. 3.1. Representations of type IIa, IIb, X, XIa and XIb are Siegel induced and given by Prop. 3.2 .

For the trivial representation $\mathbf{1}_{\mathrm{GSp}(4, F)}$ of type IVd, the parahoric restriction at $\mathscr{J}$ is clearly the trivial representation $[\mathbf{1}, \mathbf{1}]$. The representation $\rho=L\left(\nu^{3 / 2} \mathrm{St}, \nu^{-3 / 2}\right)$ of type IVc is the non-trivial constituent of the Klingen induced representation $\nu^{2} \times \nu^{-1} \mathbf{1}_{\mathrm{GSp}(2, F)}$ [6. (2.9)]. By exactness and Prop. 3.1. its parahoric restriction is $\mathbf{r}_{\mathscr{J}}(\rho)=[\mathrm{St}, \mathbf{1}]+$ $[\mathbf{1}, \mathrm{St}]+[\mathbf{1}, \mathbf{1}]$. By the analogous argument with Prop. 3.2, the parahoric restriction for representations of type IVa and IVb is clear.

For $\rho=L\left(\nu^{1 / 2} \xi \mathrm{St}, \nu^{-1 / 2} \xi\right)$ of type $\mathrm{Vc}$ with an unramified quadratic character $\xi=\xi_{u}$, the parahoric restriction $\mathbf{r}_{\mathscr{J}}(\rho)$ is contained in $\mathbf{r}_{\mathscr{J}}\left(\nu^{1 / 2} \xi_{u} \mathbf{1}_{\mathrm{GL}(2)} \rtimes \nu^{-1 / 2}\right)=[1 \times 1,1 \times 1]$. 
By [7, Thm. 3.30], $\mathbf{r}_{\mathscr{J}}(\rho)$ has a generic subquotient, which must be [St, St]. There is exactly one further constituent in $\mathbf{r}_{\mathscr{J}}(\rho)$, because the Klingen parahoric restriction $\mathbf{r}_{\mathscr{Q}}(\rho)$ contains two constituents by Table 4. By Lemma 3.4, this can only be $[\mathbf{1}, \mathbf{1}]$. For tamely ramified quadratic character $\xi=\xi_{t}$, the parahoric restriction $\mathbf{r}_{\mathscr{J}}(\rho)$ is given by [7, §3.3.3]. For types $\mathrm{Va}, \mathrm{Vb}$ and $\mathrm{Vd}$ the result is clear by Prop. 3.2 and exactness.

The representation $\rho=\tau\left(S, \nu^{-1 / 2}\right)$ of type VIa is a constituent of the Klingen induced representation $\nu^{1 / 2} \mathrm{St}_{\mathrm{GL}(2, F)} \rtimes \nu^{-1 / 2}$ and of the Siegel induced representation $1 \rtimes \mathrm{St}_{\mathrm{GSp}(2, F)}$ [6. (2.11)]. By Prop. 3.1 and 3.2, the parahoric restriction at $\mathscr{J}$ is a subquotient of $[\mathrm{St}, \mathrm{St}]+[\mathbf{1}, \mathrm{St}]+[\mathrm{St}, \mathbf{1}]$. The Klingen parahoric restriction $\mathbf{r}_{\mathscr{Q}}(\rho)$ has three irreducible constituents, so $[3]$ implies $\mathbf{r}_{\mathscr{J}}(\rho) \cong[\mathrm{St}, \mathrm{St}]+[\mathbf{1}, \mathrm{St}]+[\mathrm{St}, \mathbf{1}]$. For types VIb, VIc and VId the result is clear by exactness.

Representations of type VIII are irreducible subquotients of $1 \rtimes \pi$ with $\pi$ of depth zero. Their paramodular restriction is either $[\widetilde{\pi}, \mathbf{1}]+[\mathbf{1}, \widetilde{\pi}]$ or $[\widetilde{\pi}, \mathrm{St}]+[\mathrm{St}, \widetilde{\pi}]$ by Prop. 3.1 and Lemma 3.4. The rest of the argument is analogous to the hyperspecial case: By [7, Cor. 4.23], the character value of

$$
\mathbf{r}_{\mathscr{J}}(\tau(S, \pi)-\tau(T, \pi))
$$

on the conjugacy class stably conjugate to $\left(\operatorname{diag}\left(\alpha \beta, \alpha^{q} \beta^{q}\right), \operatorname{diag}\left(\alpha \beta^{q}, \alpha^{q} \beta\right)\right)$ for $\alpha, \beta \in \mathbb{F}_{q^{2}}^{\times}$ with $\alpha, \beta, \alpha \beta, \alpha \beta^{q} \notin \mathbb{F}_{q}^{\times}$is given by

$$
\begin{gathered}
-2\left(\Lambda(\alpha)+\Lambda\left(\alpha^{q}\right)\right)\left(\Lambda(\beta)+\Lambda\left(\beta^{q}\right)\right) \\
=2\left(-\Lambda(\alpha \beta)-\Lambda^{q}(\alpha \beta)\right)+2\left(-\Lambda\left(\alpha \beta^{q}\right)-\Lambda^{q}\left(\alpha \beta^{q}\right)\right) .
\end{gathered}
$$

This implies $\mathbf{r}_{\mathscr{J}}(\tau(T, \pi))=[\widetilde{\pi}, \mathrm{St}]+[\mathrm{St}, \widetilde{\pi}]$ and $\mathbf{r}_{\mathscr{J}}(\tau(S, \pi))=[\tilde{\pi}, \mathbf{1}]+[\mathbf{1}, \widetilde{\pi}]$.

For type IX, see [7, §3.3.2].

\section{Tables}

The irreducible admissible representations of $\operatorname{GSp}(4, F)$ have been classified by Sally and Tadić [8]. We use the notation of Roberts and Schmidt [6].

For $i=0,1,2$ let $\mu_{i}: F^{\times} \rightarrow \mathbb{C}^{\times}$be tamely ramified or unramified characters. Let $\pi$ be an arbitrary cuspidal irreducible admissible representation of $\mathrm{GL}(2, F)$ of depth zero. Its hyperspecial restriction $\mathbf{r}_{\mathrm{GL}(2, \mathfrak{o})}(\pi)=\widetilde{\pi}=\widetilde{\pi}_{\Lambda}$ is an irreducible cuspidal representation of $\mathrm{GL}(2, q)$. Up to a sign it is the Deligne-Lusztig representation attached to a character $\Lambda$ of $\mathbb{F}_{q^{2}}^{\times}$in general position, i.e. $\Lambda \neq \Lambda^{q}$. The contragredient of $\widetilde{\pi}$ is denoted $\widetilde{\pi}^{\vee}$.

The non-trivial unramified quadratic character of $F^{\times}$is denoted $\xi_{u}$. For odd $q$ let $\xi_{t}$ be one of the two tamely ramified quadratic characters which reduce to the non-trivial quadratic character $\lambda_{0}$ of $\mathbb{F}_{q}^{\times}$. For even $q$ there is no tamely ramified quadratic character.

Table 3. For even $q$, irreducible characters of $\operatorname{Sp}(4, q)$ have been classified by Enomoto [4]. By the isomorphism $\operatorname{Sp}(4, q) \times \mathrm{GL}(1, q) \cong \mathrm{GSp}(4, q),(x, t) \mapsto t \cdot x$, the irreducible 
characters of $\operatorname{GSp}(4, q)$ can be classified in terms of their restriction to $\operatorname{Sp}(4, q)$ and their central character. Fix a generator $\hat{\theta}$ of the cyclic character group of $\mathbb{F}_{q^{2}}^{\times}$and denote its restrictions to $\mathbb{F}_{q}^{\times}$by $\hat{\gamma}$ and to $\mathbb{F}_{q^{2}}^{\times}[q+1]$ by $\hat{\eta}$, respectively. Let $k_{i} \in \mathbb{Z} /(q-1) \mathbb{Z}$ be such that $\hat{\gamma}^{k_{i}}=\widetilde{\mu_{i}}$. Let $l \in \mathbb{Z} /\left(q^{2}-1\right) \mathbb{Z}$ be such that $\Lambda=\hat{\theta}^{l}$ and let $l^{\prime}$ be the image of $l$ under the canonical projection $\mathbb{Z} /\left(q^{2}-1\right) \mathbb{Z} \rightarrow \mathbb{Z} /(q+1) \mathbb{Z}$ so that the restriction of $\Lambda$ to $\mathbb{F}_{q^{2}}^{\times}[q+1]$ is $\hat{\eta}^{\prime}$. If $(q+1) l=0$, there is a unique preimage $l^{\prime \prime}$ of $l$ under the canonical injection $\mathbb{Z} /(q+1) \mathbb{Z} \hookrightarrow \mathbb{Z} /\left(q^{2}-1\right) \mathbb{Z}$.

For odd $q$, irreducible characters of $\operatorname{GSp}(4, q)$ have been classified by Shinoda [9]. A character $\Lambda$ of $\mathbb{F}_{q^{2}}^{\times}$with $\Lambda^{q+1}=1$ factors over a character $\omega_{\Lambda}$ of $\mathbb{F}_{q^{2}}^{\times}[q+1]$ via $\Lambda(\alpha)=$ $\omega_{\Lambda}\left(\alpha^{q-1}\right)$. If $\Lambda^{q-1}$ is the quadratic character $\Lambda_{0}$ of $\mathbb{F}_{q^{2}}^{\times}$, there is a unique character $\lambda^{\prime}$ of $\mathbb{F}_{q^{2}}^{\times}[2(q-1)]$ with $\Lambda(\alpha)=\lambda^{\prime}\left(\alpha^{(q+1) / 2}\right)$.

Table 4. The trivial and the Steinberg representation of $\operatorname{GL}(2, q)$ are denoted $\mathbf{1}$ and St, respectively. The parabolic induction of the character $\mu_{1} \otimes \mu_{2}$ of the standard torus is denoted $\mu_{1} \times \mu_{2}$. For typographical reasons, we write

$$
\begin{aligned}
& A\left(\widetilde{\mu_{1}}, \widetilde{\mu_{2}}, \widetilde{\mu_{0}}\right)=\widetilde{\mu_{1}} \nabla \widetilde{\mu_{2}} \nabla \widetilde{\mu_{0}}+\widetilde{\mu_{1}} \nabla \widetilde{\mu_{2}}-1 \nabla \widetilde{\mu_{2}} \widetilde{\mu_{0}}+\widetilde{\mu_{1}}-1 \nabla \widetilde{\mu_{2}} \nabla \widetilde{\mu_{1}} \widetilde{\mu_{0}} \\
& +{\widetilde{\mu_{1}}}^{-1} \otimes{\widetilde{\mu_{2}}}^{-1} \otimes \widetilde{\mu_{1}} \widetilde{\mu_{2}} \widetilde{\mu_{0}}, \\
& B\left(\widetilde{\mu_{1}}, \widetilde{\mu_{2}}, \widetilde{\mu_{0}}\right)=\widetilde{\mu_{1}} \otimes\left(\widetilde{\mu_{2}} \rtimes \widetilde{\mu_{0}}\right)+{\widetilde{\mu_{1}}}^{-1} \otimes\left(\widetilde{\mu_{2}} \rtimes \widetilde{\mu_{1}} \widetilde{\mu_{0}}\right), \\
& C\left(\widetilde{\mu_{1}}, \widetilde{\mu_{2}}, \widetilde{\mu_{0}}\right)=\left(\widetilde{\mu_{1}} \times \widetilde{\mu_{2}}\right) \otimes \widetilde{\mu_{0}}+\left({\widetilde{\mu_{1}}}^{-1} \times \widetilde{\mu_{2}}\right) \otimes \widetilde{\mu_{1}} \widetilde{\mu_{0}} \text {. }
\end{aligned}
$$

Table 5. We call a representation of $\left(\operatorname{GL}(2, q)^{2}\right)^{0}=\left\{(a, b) \in \operatorname{GL}(2, q)^{2} \mid \operatorname{det} a=\operatorname{det} b\right\}$ generic if it is generic with respect to the unipotent character

$$
\left(\left(\begin{array}{ll}
1 & x \\
& 1
\end{array}\right),\left(\begin{array}{ll}
1 & y \\
& 1
\end{array}\right)\right) \mapsto \widetilde{\psi}(x+y) .
$$

This does not depend on the choice of the non-trivial additive character $\widetilde{\psi}$ of $\mathbb{F}_{q}$. The irreducible representations of $\operatorname{GL}(2, q) \times \operatorname{GL}(2, q)$ are $\sigma_{1} \otimes \sigma_{2}$ for irreducible representations $\sigma_{i}$ of $\mathrm{GL}(2, q)$. We denote the restriction of $\sigma_{1} \otimes \sigma_{2}$ to $\left(\mathrm{GL}(2, q)^{2}\right)^{0}$ by $\left[\sigma_{1}, \sigma_{2}\right]$. This restriction is irreducible unless $\lambda_{0} \sigma_{1} \cong \sigma_{1}$ and $\lambda_{0} \sigma_{2} \cong \sigma_{2}$ for the non-trivial quadratic character $\lambda_{0}$ of $\mathbb{F}_{q}^{\times}$, then it splits into an equidimensional direct sum of a generic constituent $\left[\sigma_{1}, \sigma_{2}\right]_{+}$and a non-generic constituent $\left[\sigma_{1}, \sigma_{2}\right]_{-}$. These are all the irreducible representations of $\left(\operatorname{GL}(2, q)^{2}\right)^{0}$. The twist of a representation $\sigma$ of $\left(\operatorname{GL}(2, q)^{2}\right)^{0}$ by a character $\tilde{\mu}$ of $\mathbb{F}_{q}^{\times}$is defined by

$$
\widetilde{\mu}\left[\sigma_{1}, \sigma_{2}\right]=\left[(\widetilde{\mu} \circ \operatorname{det}) \otimes \sigma_{1}, \sigma_{2}\right]=\left[\sigma_{1},(\widetilde{\mu} \circ \operatorname{det}) \otimes \sigma_{2}\right] .
$$

Acknowledgements. The author wants to express his gratitude to R. Weissauer and U. Weselmann for valuable discussions. 


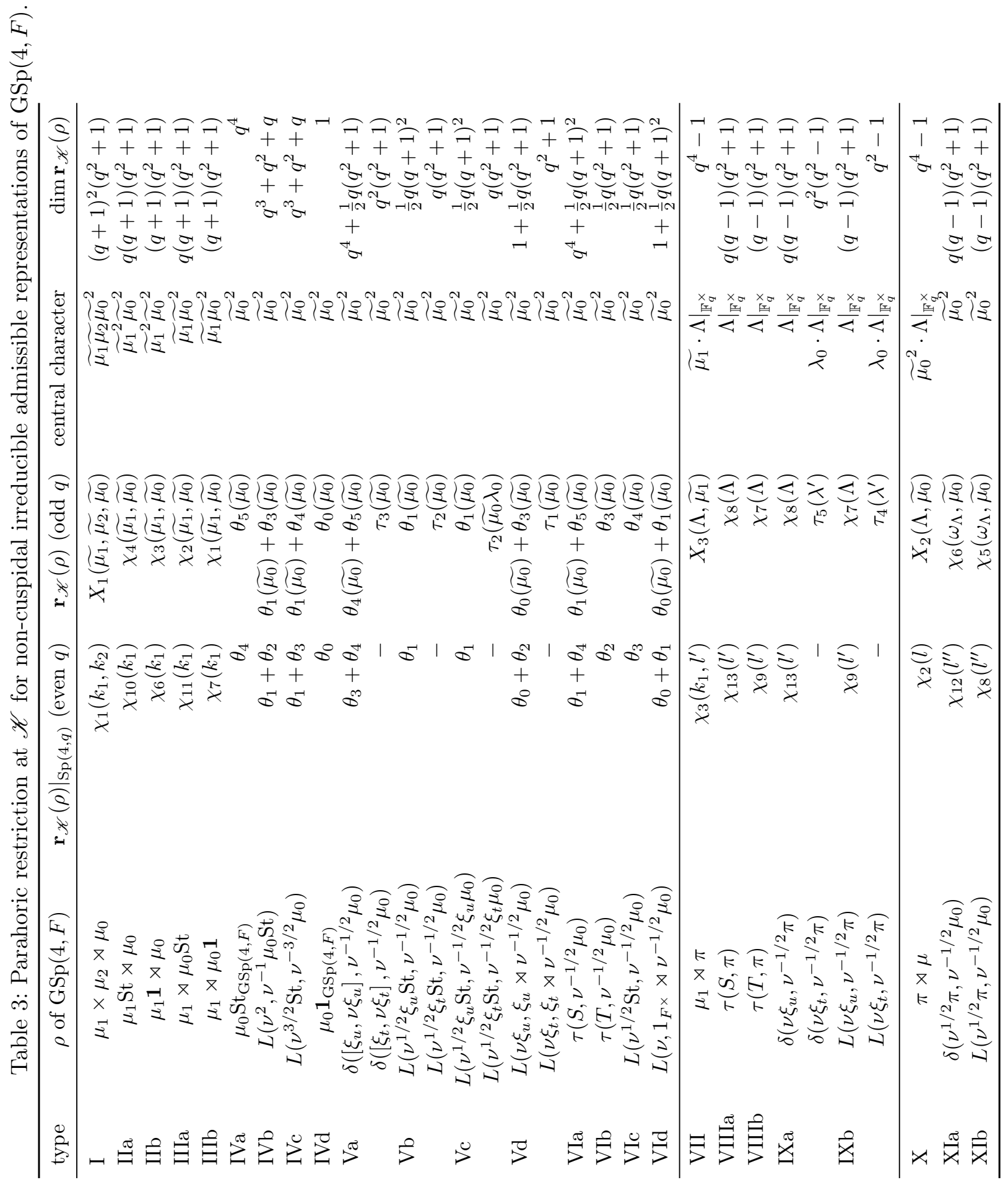




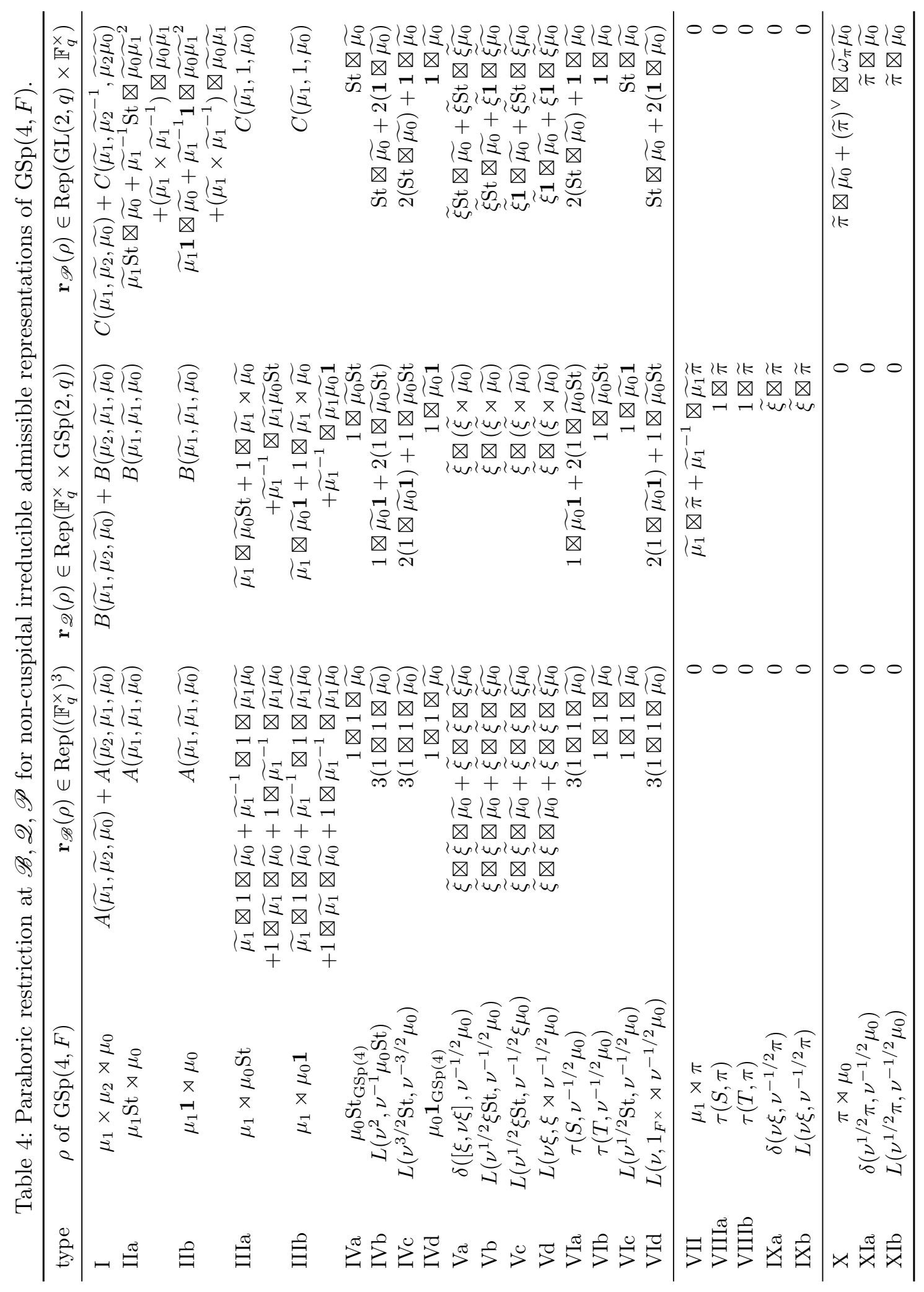


Table 5: Parahoric restriction at $\mathscr{J}$ for non-cuspidal irreducible admissible representations of $\operatorname{GSp}(4, F)$. The index is determined by $\xi_{t}(\varpi)= \pm 1$.

\begin{tabular}{|c|c|c|c|}
\hline type & $\rho$ of $\operatorname{GSp}(4, F)$ & $\mathbf{r}_{\mathscr{J}}(\rho) \in \operatorname{Rep}\left(\left(\operatorname{GL}(2, q)^{2}\right)^{0}\right)$ & $\operatorname{dim} \mathbf{r}_{\mathscr{J}}(\rho)$ \\
\hline I & $\mu_{1} \times \mu_{2} \rtimes \mu_{0}$ & $\widetilde{\mu_{0}}\left[1 \times \widetilde{\mu_{1}}, 1 \times \widetilde{\mu_{2}}\right]+\widetilde{\mu_{0}}\left[1 \times \widetilde{\mu_{2}}, 1 \times \widetilde{\mu_{1}}\right]$ & $2(q+1)^{2}$ \\
\hline IIa & $\mu_{1} \mathrm{St} \rtimes \mu_{0}$ & $\widetilde{\mu_{0}}\left[1 \times \widetilde{\mu_{1}}, 1 \times \widehat{\mu_{1}}\right]$ & $(q+1)^{2}$ \\
\hline IIb & $\mu_{1} \mathbf{1} \rtimes \mu_{0}$ & $\widetilde{\mu_{0}}\left[1 \times \widetilde{\mu_{1}}, 1 \times \widetilde{\mu_{1}}\right]$ & $(q+1)^{2}$ \\
\hline IIIa & $\mu_{1} \rtimes \mu_{0} \mathrm{St}$ & $\widetilde{\mu_{0}}\left[1 \times \widetilde{\mu_{1}}, \mathrm{St}\right]+\widetilde{\mu_{0}}\left[\mathrm{St}, 1 \times \widetilde{\mu_{1}}\right]$ & $2 q(q+1)$ \\
\hline IIIb & $\mu_{1} \rtimes \mu_{0} \mathbf{1}$ & $\widetilde{\mu_{0}}\left[1 \times \widetilde{\mu_{1}}, \mathbf{1}\right]+\widetilde{\mu_{0}}\left[\mathbf{1}, 1 \times \widetilde{\mu_{1}}\right]$ & $2(q+1)$ \\
\hline IVa & $\mu_{0} \mathrm{St}_{\mathrm{GSp}(4, F)}$ & $\widetilde{\mu_{0}}[\mathrm{St}, \mathrm{St}]$ & $q^{2}$ \\
\hline $\mathrm{IVb}$ & $L\left(\nu^{2}, \nu^{-1} \mu_{0} \mathrm{St}\right)$ & $\widetilde{\mu_{0}}[\mathrm{St}, \mathrm{St}]+\widetilde{\mu_{0}}[\mathbf{1}, \mathrm{St}]+\widetilde{\mu_{0}}[\mathrm{St}, \mathbf{1}]$ & $q^{2}+2 q$ \\
\hline IVc & $L\left(\nu^{3 / 2} \mathrm{St}, \nu^{-3 / 2} \mu_{0}\right)$ & $\widetilde{\mu_{0}}[\mathbf{1}, \mathbf{1}]+\widetilde{\mu_{0}}[\mathbf{1}, \mathrm{St}]+\widetilde{\mu_{0}}[\mathrm{St}, \mathbf{1}]$ & $2 q+1$ \\
\hline IVd & $\mu_{0} \mathbf{1}_{\mathrm{GSp}(4, F)}$ & $\widetilde{\mu_{0}}[\mathbf{1}, \mathbf{1}]$ & 1 \\
\hline \multirow[t]{2}{*}{ Va } & $\delta\left(\left[\xi_{u}, \nu \xi_{u}\right], \nu^{-1 / 2} \mu_{0}\right)$ & $\widetilde{\mu_{0}}[\mathbf{1}, \mathrm{St}]+\widetilde{\mu_{0}}[\mathrm{St}, \mathbf{1}]$ & $2 q$ \\
\hline & $\delta\left(\left[\xi_{t}, \nu \xi_{t}\right], \nu^{-1 / 2} \mu_{0}\right)$ & $\widetilde{\mu_{0}}\left[1 \times \lambda_{0}, 1 \times \lambda_{0}\right]_{ \pm}$ & $(q+1)^{2} / 2$ \\
\hline \multirow[t]{2}{*}{$\mathrm{Vb}$} & $L\left(\nu^{1 / 2} \xi_{u} \mathrm{St}, \nu^{-1 / 2} \mu_{0}\right)$ & $\widetilde{\mu_{0}}[\mathbf{1}, \mathbf{1}]+\widetilde{\mu_{0}}[\mathrm{St}, \mathrm{St}]$ & $q^{2}+1$ \\
\hline & $L\left(\nu^{1 / 2} \xi_{t} \mathrm{St}, \nu^{-1 / 2} \mu_{0}\right)$ & $\widetilde{\mu_{0}}\left[1 \times \lambda_{0}, 1 \times \lambda_{0}\right]_{\mp}$ & $(q+1)^{2} / 2$ \\
\hline \multirow[t]{2}{*}{ Vc } & $L\left(\nu^{1 / 2} \xi_{u} \mathrm{St}, \nu^{-1 / 2} \xi_{u} \mu_{0}\right)$ & $\widetilde{\mu_{0}}[\mathbf{1}, \mathbf{1}]+\widetilde{\mu_{0}}[\mathrm{St}, \mathrm{St}]$ & $q^{2}+1$ \\
\hline & $L\left(\nu^{1 / 2} \xi_{t} \mathrm{St}, \nu^{-1 / 2} \xi_{t} \mu_{0}\right)$ & $\widetilde{\mu_{0}}\left[1 \times \lambda_{0}, 1 \times \lambda_{0}\right]_{\mp}$ & $(q+1)^{2} / 2$ \\
\hline \multirow[t]{2}{*}{$\mathrm{Vd}$} & $L\left(\nu \xi_{u}, \xi_{u} \rtimes \nu^{-1 / 2} \mu_{0}\right)$ & $\widetilde{\mu_{0}}[\mathbf{1}, \mathrm{St}]+\widetilde{\mu_{0}}[\mathrm{St}, \mathbf{1}]$ & \\
\hline & $L\left(\nu \xi_{t}, \xi_{t} \rtimes \nu^{-1 / 2} \mu_{0}\right)$ & $\widetilde{\mu_{0}}\left[1 \times \lambda_{0}, 1 \times \lambda_{0}\right]_{ \pm}$ & $(q+1)^{2} / 2$ \\
\hline VIa & $\tau\left(S, \nu^{-1 / 2} \mu_{0}\right)$ & $\widetilde{\mu_{0}}[\mathrm{St}, \mathrm{St}]+\widetilde{\mu_{0}}[\mathrm{St}, \mathbf{1}]+\widetilde{\mu_{0}}[\mathbf{1}, \mathrm{St}]$ & $q^{2}+2 q$ \\
\hline VIb & $\tau\left(T, \nu^{-1 / 2} \mu_{0}\right)$ & $\dot{\mu_{0}}[\mathrm{St}, \mathrm{St}]$ & $q^{2}$ \\
\hline VIc & $L\left(\nu^{1 / 2} \mathrm{St}, \nu^{-1 / 2} \mu_{0}\right)$ & $\tilde{\mu_{0}}[\mathbf{1}, \mathbf{1}]$ & 1 \\
\hline VId & $L\left(\nu, 1_{F \times} \rtimes \nu^{-1 / 2} \mu_{0}\right)$ & $\widetilde{\mu_{0}}[\mathbf{1}, \mathbf{1}]+\widetilde{\mu_{0}}[\mathrm{St}, \mathbf{1}]+\widetilde{\mu_{0}}[\mathbf{1}, \mathrm{St}]$ & $2 q+1$ \\
\hline VII & $\mu_{1} \rtimes \pi$ & {$\left[1 \times \widetilde{\mu_{1}}, \tilde{\pi}\right]+\left[\widetilde{\pi}, 1 \times \widetilde{\mu_{1}}\right]$} & $2\left(q^{2}-1\right)$ \\
\hline VIIIa & $\tau(S, \pi)$ & {$[\mathbf{1}, \widetilde{\pi}]+[\widetilde{\pi}, \mathbf{1}]$} & $2(q-1)$ \\
\hline VIIIIb & $\tau(T, \pi)$ & {$[\mathrm{St}, \widetilde{\pi}]+[\widetilde{\pi}, \mathrm{St}]$} & $2(q-1) q$ \\
\hline \multirow[t]{2}{*}{ IXa } & $\delta\left(\nu \xi_{u}, \nu^{-1 / 2} \pi\right)$ & {$[\mathrm{St}, \tilde{\pi}]+[\tilde{\pi}, \mathrm{St}]$} & $2(q-1) q$ \\
\hline & $\delta\left(\nu \xi_{t}, \nu^{-1 / 2} \pi\right)$ & {$\left[\widetilde{\pi}, 1 \times \lambda_{0}\right]_{\mp}+\left[1 \times \lambda_{0}, \widetilde{\pi}\right]_{\mp}$} & $q^{2}-1$ \\
\hline \multirow[t]{2}{*}{$\mathrm{IXb}$} & $L\left(\nu \xi_{u}, \nu^{-1 / 2} \pi\right)$ & {$[\mathbf{1}, \widetilde{\pi}]+[\widetilde{\pi}, \mathbf{1}]$} & $2(q-1)$ \\
\hline & $L\left(\nu \xi_{t}, \nu^{-1 / 2} \pi\right)$ & {$\left[\tilde{\pi}, 1 \times \lambda_{0}\right]_{ \pm}+\left[1 \times \lambda_{0}, \widetilde{\pi}\right]_{ \pm}$} & $q^{2}-1$ \\
\hline $\mathrm{X}$ & $\pi \rtimes \mu_{0}$ & 0 & 0 \\
\hline XIa & $\delta\left(\nu^{1 / 2} \pi, \nu^{-1 / 2} \mu_{0}\right)$ & 0 & 0 \\
\hline $\mathrm{XIb}$ & $L\left(\nu^{1 / 2} \pi, \nu^{-1 / 2} \mu_{0}\right)$ & 0 & 0 \\
\hline
\end{tabular}




\section{References}

[1] Borel, A.: Admissible Representations of a Semi-Simple Group over a Local Field with Vectors Fixed under an Iwahori Subgroup. Inv. Math. 35, 233-259 (1976)

[2] Breeding-Allison, J.: Irreducible Characters of $G S p\left(4, \mathbb{F}_{q}\right)$. Ramanujan J. 36(3), 305-354 (2015)

[3] Bushnell, C.J., Henniart, G.: The Local Langlands Conjecture for GL(2). Grundlehren der Mathematischen Wissenschaften. Springer (2006)

[4] Enomoto, H.: The characters of the finite symplectic group $S p(4, q), q=2^{f}$. Osaka J. Math. 9, 75-94 (1972)

[5] Moy, A., Prasad, G.: Jacquet functors and unrefined minimal K-types. Comment. Math. Helvetici 71, 98-121 (1996)

[6] Roberts, B., Schmidt, R.: Local Newforms for GSp(4), Lecture Notes in Mathematics, vol. 1918, 1 edn. Springer (2007)

[7] Rösner, M.: Parahoric restriction for $\operatorname{GSp}(4)$ and the inner cohomology of Siegel modular threefolds. Ph.D. thesis, University of Heidelberg (2016). doi:10.11588/heidok.00021401

[8] Sally, P., Tadić, M.: Induced Representations and classifications for $G S p(2, F)$ and $S p(2, F)$. Mémoires Société Mathématique de France 52, 75-133 (1994)

[9] Shinoda, K.I.: The characters of the finite conformal symplectic group $C S p(4, q)$. Communications in Algebra 10(13), 1369-1419 (1982)

[10] Tadić, M.: On Jacquet Modules of Induced representations of p-adic symplectic groups. In: Harmonic Analysis on Reductive Groups, Progress in Mathematics, vol. 101, pp. 305-314. Birkhäuser (1991)

[11] Vignéras, M.F.: Représentations $l$-modulaires d'un groupe réductif $p$-adique avec $l \neq p$, Progress in Mathematics, vol. 137. Birkhäuser (1996)

[12] Vignéras, M.F.: Irreducible Modular Representations of a reductive $p$-adic group and simple Modules for Hecke Algebras. In: C. Casacuberta, et al. (eds.) European Congress of Mathematics, Barcelona, Progress in Mathematics, vol. 201, pp. 117133. Birkhäuser (2001)

[13] Vignéras, M.F.: Schur Algebras of reductive p-adic groups. Duke Math. J. 116(1), $35-75(2003)$

[14] Weissauer, R.: Endoscopy for GSp(4) and the Cohomology of Siegel Modular Threefolds, Lecture notes in Mathematics, vol. 1968. Springer (2009) 